\title{
The Crisis Management of Enterprise's Collective Brain Drain
}

\author{
Chunli Yuan \& Chunying Zhang \\ School of Business Administration, Tianjin Polytechnic University \\ Tianjin 300387, China \\ E-mail: yuanchunli666@163.com, zhchy169@163.com
}

\begin{abstract}
This paper take enterprise's collective brain drain as the research object, by using of questionnaire method, historical data, experts consultation method, and other methods of construct the collective brain drain of forewarning indicators, and then use the alternative ranking method to determine and evaluate comprehensively system of indicators to the weight. By constructing the forewarning system to real-time monitor, identify, diagnose and evaluate the trend of enterprise's collective brain drain and on this basis to pre-control, the prior development of corrective measures, the crisis will be resolved in the embryonic stage. Therefore, constructing the forewarning system of enterprise's collective brain drain for the long-term stable development of enterprises has important practical significance.
\end{abstract}

Keywords: Core talent, Collective brain drain, Crisis, Forewarning management, Pre-control

\section{Introduction:}

The brain drain contains individual brain drain and collective brain drain and compared to the individual brain drain, collective brain drain does greater harm to the original enterprises. Collective brain drain is not just only a few people leave the corporation ,but it means the lost of the issue of corporate management skills, core technologies, marketing strategies and ideas, goods supply and marketing channels, customer information and so on. In addition, it will also affect the staff's mood and greatly dampened the morale of the overall enterprise; the more seriously damaged the company's image. Collective brain drain has become the most fatal injuries to enterprise management. In overseas, the research of enterprise's forewarning management is mainly empirical and the contents focus on enterprise-level functions, such as early warning of financial, marketing, but the study of forewarning the loss of staff in the management and building a forewarning principle of unity in the forewarning system is scare.

In china, currently on the brain drain of many causes for the study of countermeasures, such as compensations to keep staff, establish psychological contract, organizational commitment, and so on. Forewarning and pre-control system can effectively make up for the study's defect of the sudden loss of talent and unpredictability. Recently enterprises brain drain has the biggest characteristic of the core talent of "collective resignation", and the trend has intensified. In 1995 when "little overlord" is at the peak, Duan Yongping as manager led almost all middle-level managers of collective resignation, leading to "little overlord" history. Founder, Executive Director Zhou Xianfeng led more than 30 PC technology backbone of joining into Hisense Digital. These incidents have caused great uproar in the industry. Data show that: a staff of 2,300 dollars for the replacement cost in the United States, this is tangible. Intangible capital including: available in the old value of the experience of the staff loss, new employees not familiar with the work environment due to the impact of his skills to play the normal capacity loss, and enterprises in order to guide the new staff and the loss of human and material. From the economics point of view, after control is not better than course control and course control is not better than advance control. Forewarning management belongs to advance control in the control theory, it is through the detection and identification of the factors leading to the incident control the outcome of the case, more effective control over the course control. "Cause analysis - forewarning management - pre-control measures" is as the main line for the loss of enterprise's collective brain drain crisis, with a view to avoiding enterprise from collective brain drain.

\section{Cause Analysis}

Since the establishment of market economic system and improvement the macro environment or career change in policy, update the concept of talent flow, social changes in employment patterns, the emergence of new occupation and the international environmental factors make more jobs and careers of freedom; Impact on the flow of talent have played a role in encouraging and promoting, greatly increases the possibility of brain drain. The main factors of enterprise's collective brain drain including: the external environmental factors, organizational factors, personal factors.

\subsection{External Environmental Factors}

With the advent of the era of knowledge economy, knowledge workers normally have the pursuit of personal 
achievements desire. And the knowledge work's most outstanding characteristics is team model, when a team members to leave their team and his personal values will become very small, single individuals can not be based on the market. Once the core figure left in the team he is bound to take the whole team. Phenomenon of collective loss is the inevitable product of the times. Enterprises to foster a team need to cost a lot of manpower, material and financial resources. Particularly in the high-tech enterprises, the contribution of the individual employees is getting smaller and smaller, and the team's value is getting bigger and bigger. Otherwise, digging a team is more effective than digging individual, which can save a lot of training costs, research and development costs and marketing costs. So, many enterprises use of head hunter dig the mature rival team.

\subsection{Organizational Factors}

To organization aspect, the basic reason of causing excellent teams' loss is that corporate management issues. The main problems are that: high-quality managers, staff incentive mechanism is inadequate and fails to establish an effective evaluation system, the lack of a reasonable salary structure; the team could not build for the long-term development planning and corporate culture. In addition, the company's high-level personnel changes in the overall development strategy and corporate adjustments, making the company's management concept and corporate culture a lot of changes, and this change is unacceptable to the existing team, so team chose to leave.

\subsection{Personal Factors}

The excellent talent wants to achieve through the development of their self-worth, broaden development space and reasonable career planning. When they feel that companies unable to raise the value of their own space and can not learn new skills, lacking of training and growth opportunities they will choose to leave. In addition, a team member agree the way of the core leader with its management conception, work atmosphere, the way very much, the core figure's left could lead to the entire team to follow.

\section{Forewarning Management}

At this stage the construction of forewarning indicator system, including the study of forewarning indicators of system construction, crisis assessment and issue a final warning.

\subsection{Construction of the collective brain drain forewarning indicator system}

Through the questionnaire method, historical data and experts' consultation method in follow SMART principles: Specific, measurable, action-oriented, realistic, and time and resource constrained based on the principles of design indicator system. Although the collective loss is the loss of the individual, but it is not a simple superposition of individual loss. The total index should include: the space of team development, the satisfaction of team compensation and benefits, the harmony degree of team and corporate, the behaviour of team changes, the competitiveness of industry. In accordance with the actual situation and the characteristics of the enterprises of the sector indicators screening and thinning, then the establish second index, third or even fourth index, fifth index, so as to make the minimum index system to reflect the most complete collection of information.

\subsection{Construction of the crisis evaluation system}

Taking into account cost factors and operability, in this process using two-factor evaluation of the crisis assessment and forewarning warning condition. Taking into account cost factors and operability, in this process using two-factor evaluation of the crisis assessment and forewarning warning condition. Risk index is the product of the probability of crisis and the crisis damage degree, according to damage index degree, through historical data and expert's consultation method to determine their collective brain drain damage degree, and send out warnings. First, according to the enterprise's historical data and experts' consultation to determine probability of all the indicators (see table 1). Then, by alternative ranking method identified by the index system of indicators in each damage degree evaluation, that is: choose two indexes which will cause the greatest and lest collective brain drain crisis, and then again in the remaining indicators in which will cause the greatest and lest collective brain drain crisis. In the same way, determine all the indicators of the crisis damage degree in order, and use of experts consultation to determine the weight (see table 2). At last, determine the risk index of collective brain drain crisis.

\subsection{Determine forewarning degree}

Before the collective brain drain happened, there is always some abnormal behaviour.

Sign 1: Team leaders or the core figures that have greater influence on the team present more arrogant and more frequent contacts with the outside world.

Sign 2: Team activities present more frequent and secret characteristic, in the other hand team members are too close contacts with the outside world.

Sign 3: Team complaint increased and ask for to higher requirements for enterprises.

Sign 4: The enthusiasm of team work decreased significantly and the working efficiency became poor. 
Sign 5: Enterprise suddenly appeared a number of gangs, and mainly to a person often entertained some staff members. This situation is likely to be the leave prelude.

Sign 6: While concentrating stage, phenomenon of a group of the quarrel, the commentary justified and phenomenon not have been, but suddenly burst, with centralized and explosive characteristics.

According to the sign of warning changes, in the light of crisis evaluation criteria and combined with actual or future to make suitable amendments to get the severity of the situation by warning degrees. After determining the forewarning indicators and calculated forewarning condition's degree, establish forewarning signal system, which is similar to that adopted a set of traffic control signal red, yellow and green logo, this group of indicators reflected in collective brain drain situation in a different signal. Finally, by analysis the changes in signal, to determine the future of corporate personnel collective brain drain of the trend or the extent of the loss.

\section{Pre-control Measures}

Following the enterprise's collective brain drain forewarning management we can get the extent and the trend of the collective brain drain. Then the task is to take the positive effectual pre-control measures to eliminate or control the spread of problem development.

\subsection{Information Communication}

Human Resources Department in support of high-ranking executives, around the content of enterprise's collective brain drain indicator system, established specialized information channels to meet the specific needs and to ensure smooth flow of information. Then, consisting the Expert Group by the Human Resources Department, heads of other departments and external experts the, to process the collection of information. According to results of forewarning analysis and the past experience design different risky degree, operational emergency project and handling ways to approach of the crisis preparedness strategy base. Such as compensation incentive preparedness base, Cost Constraint preparedness base, legal restraint preparedness base, knowledge management preparedness base.

\subsection{Preparedness Determination and Implementation}

According to warning condition categories, divided into two parts. The first part is storing the conventional case to cope with various crises, it can automatically output a number of countermeasures according to the nature and type of situation. The second part is dealing with non-conventional expert's consultation system, using the Delphi method to complete the consultation exercise to get countermeasures, and record storage strategy for future calls. Finally, preparedness to compare, evaluate, select the best plan. In access to high-level managers' support, from the Human Resources Department to make out an implementation plan, implement pre-control preparedness under plans and steps; really achieve the objective of crisis prevention.

\section{Conclusion}

The establishment of a comprehensive and sensitive collective brain drain crisis system, timely detection endanger the survival and development of enterprise's collective brain drain, forecast its development trends, assess the damage ,promptly issued a warning to the crisis and timely issue warning to the crisis, so that enterprises ahead of crisis intervention, early to take effective measures to prevent the crisis in the implementation of pre-control, will solve the problem in the embryonic stage.

\section{References}

Basak S, Shapiro A. (2001). Value-at-Risk-based Risk Management: optimal policies and asset price. The Review of Financial Studies. 2:371 409.

Hellman, C.M.A. (1997). Job satisfaction and intent to leave. Journal of Social Psychology. 137(6): 677 689.

Liu Ping \& Zhang Chunying. (2006). The Establishment of Core Talent Crisis Forewarning System. Chinese Talents, 6 (In Chinese).

Philips, J.D. (1990). The price tag on turnover. Personnel Journal. 6:58 61. 
Table 1.

\begin{tabular}{lccccccc}
\hline index & $x_{1}$ & $x_{2}$ & $x_{3}$ & $x_{4}$ & $\cdots \cdots$ & $x_{n-1}$ & $x_{n}$ \\
\hline probability & $p_{1}$ & $p_{2}$ & $p_{3}$ & $p_{4}$ & $\cdots \cdots$ & $p_{n-1}$ & $p_{n}$ \\
\hline
\end{tabular}

Table 2.

\begin{tabular}{llllclcl}
\hline index & $x_{1}$ & $x_{2}$ & $x_{3}$ & $x_{4}$ & $\ldots \ldots$ & $x_{n-1}$ & $x_{n}$ \\
\hline damage degree & $q_{1}$ & $q_{1}$ & $q_{3}$ & $q_{4}$ & $\ldots \ldots$ & $q_{n-1}$ & $q_{n}$ \\
\hline
\end{tabular}

Calculation formula:

Probability: $\left\{p_{1}, p_{2}, p_{3}, p_{4}, \ldots \ldots, p_{n-1}, p_{n}\right\}$

Damage degree: $\left\{q_{1}, q_{1}, q_{3}, q_{4}, \ldots . ., q_{n-1}, q_{n}\right\}$

Collective brain drain crisis index: $C=\sum_{i=1}^{n} p_{i} q_{i}$ 